\title{
Trilayered Lead Chloride Perovskite Ferroelectric Affording Self- Powered Visible-Blind Ultraviolet Photodetection with Large Zero-Bias Photocurrent
}

Sasa Wang, ${ }^{\dagger}+{ }^{\ddagger}$ Lina Li, ${ }^{*},{ }^{\dagger}$ Wen Weng, ${ }^{\dagger}$ Chengmin $\mathrm{Ji}^{\dagger}{ }^{\dagger}$ Xitao Liu, ${ }^{\dagger}$ Zhihua Sun, $^{\dagger}$ Wenxiong Lin, ${ }^{\dagger}$ Maochun Hong ${ }^{\dagger}$ and Junhua Luo*, ${ }^{\dagger}$

${ }^{+}$State Key Laboratory of Structural Chemistry, Fujian Institute of Research on the Structure of Matter, Chinese Academy of Sciences, Fuzhou, Fujian 350002, China

*University of Chinese Academy of Sciences, Beijing 100049, China 


\section{EXPERIMENTAL SECTION}

Synthesis. Bulk single crystals of $\mathrm{EA}_{4} \mathrm{~Pb}_{3} \mathrm{Cl}_{10}(\mathbf{1}, \mathrm{EA}=$ ethylammonium $)$ were grown by temperature cooling method from its saturated $\mathrm{HCl}$ solution that contains stoichiometric ethylamine $(7 \mathrm{mmol})$ and $\mathrm{Pb}\left(\mathrm{CH}_{3} \mathrm{COO}\right)_{2} \cdot 3 \mathrm{H}_{2} \mathrm{O}(5 \mathrm{mmol})$. Firstly, the saturated solution was maintained $\sim 5^{\circ} \mathrm{C}$ above its saturation point for 1 day to ensure the dissolution of the solute. Then, the above mentioned solution was slowly cooling $(1 \mathrm{~K} /$ day $)$ to room temperature to obtain the large-size crystals of $\mathbf{1}$.

Measurements. Powder X-ray diffraction (PXRD) measurement was implemented using a Rigaku MiniFlex II diffractometer. The experimental PXRD pattern collected in the $2 \theta$ range of $5^{\circ}$ to $50^{\circ}$ matches well with the simulated one, confirming its phase purity (Figure S1). To investigate its thermal properties, differential scanning calorimetry (DSC) and thermogravimetric measurements were conducted on NETZSCH DSC 200 F3 DSC instrument and STA449C Thermal Analyser, respectively, with a heating/cooling rate of $10 \mathrm{~K} / \mathrm{min}$ under nitrogen atmosphere. Bulk single crystals, which covered with silver conducting paste on their surfaces, were used to measure the dielectric and ferroelectric properties of $\mathbf{1}$. The dielectric permittivity measurement was performed on a TongHui TH2828 analyser through the two-probe AC impedance method, and ferroelectric hysteresis $(P-E)$ loop was measured on a Radiant Precision Premier II using double-wave method. Variable-temperature second harmonic generation (SHG) measurement was performed on bulk crystal samples of 1 using a Nd:YAG laser $(\lambda=1064 \mathrm{~nm}, 5$ ns pulse duration, $\sim 1.6 \mathrm{MW}$ peak power, $10 \mathrm{~Hz}$ repetition rate). The $\mathrm{KH}_{2} \mathrm{PO}_{4}(\mathrm{KDP})$ was used as reference to evaluate the numerical values of the nonlinear optical coefficients. The Perkin-Elmer Lambda $900 \mathrm{UV}-\mathrm{Vis}-\mathrm{NIR}$ spectrophotometer was used to measure the absorption spectrum of $\mathbf{1}$, with the $\mathrm{BaSO}_{4}$ as the $100 \%$ reference.

Single crystal X-ray diffraction measurement of $\mathbf{1}$ was performed on a Bruker D8 diffractometer with the Mo Ka radiation at 295 and $420 \mathrm{~K}$, respectively. The data was processed by Crystalclear software package. The structures were solved by direct methods and refined by the full-matrix least-squares refinements on $F^{2}$ using SHELX-97. The detailed crystal structure data are given in Tables S1 and S2.

Calculation method. The band structure and partial density of states of $\mathbf{1}$ were calculated based on the single-crystal structure data at $295 \mathrm{~K}$ using the DFT method. In the generalized gradient approximation, the exchange and correlation effects were treated by Perdew-Burke-Ernzerh. And the norm-conserving pseudo potential was employed to depict the core-electrons interactions.

Table S1. Crystallographic Data and Structure Refinements of 1 at $295 \mathrm{~K}$ and $420 \mathrm{~K}$.

\begin{tabular}{lll}
\hline Formula sum & $\mathrm{C}_{8} \mathrm{H}_{32} \mathrm{~N}_{4} \mathrm{~Pb}_{3} \mathrm{Cl}_{10}$ & $\mathrm{C}_{8} \mathrm{H}_{32} \mathrm{~N}_{4} \mathrm{~Pb}_{3} \mathrm{Cl}_{10}$
\end{tabular}




\begin{tabular}{|c|c|c|}
\hline Formula weight $(\mathrm{g} / \mathrm{mol})$ & 1160.48 & 1160.48 \\
\hline Temperature (K) & 295 & 420 \\
\hline Crystal system & orthorhombic & tetragonal \\
\hline Space group & $C m c 2_{1}$ & $I 4 / \mathrm{mmm}$ \\
\hline$a(\AA)$ & $45.5393(19)$ & $5.7221(5)$ \\
\hline$b(\AA)$ & $8.1051(3)$ & $5.7221(5)$ \\
\hline$c(\AA)$ & $8.0343(2)$ & $45.787(6)$ \\
\hline$a(\operatorname{deg})$ & 90 & 90 \\
\hline$\beta(\operatorname{deg})$ & 90 & 90 \\
\hline$\gamma(\operatorname{deg})$ & 90 & 90 \\
\hline$V\left(\AA^{3}\right)$ & $2965.47(18)$ & $1499.2(3)$ \\
\hline$Z$ & 4 & 2 \\
\hline$D_{\text {calcd }}\left(\mathrm{g} / \mathrm{cm}^{3}\right)$ & 2.599 & 2.571 \\
\hline$\mu\left(\mathrm{mm}^{-1}\right)$ & 17.895 & 17.699 \\
\hline$F(000)$ & 2096.0 & 1054.4 \\
\hline completeness (\%) & $98.9 \%$ & $99.2 \%$ \\
\hline $\operatorname{GOF}\left(F^{2}\right)$ & 1.031 & 1.322 \\
\hline$R_{1}\left(\right.$ on $\left.F_{o}^{2}, I>2 \mathrm{~s}(I)\right)$ & 0.0375 & 0.0421 \\
\hline$w \mathrm{R}_{2}\left(\right.$ on $\left.F_{o}^{2}, I>2 \mathrm{~s}(I)\right)$ & 0.0991 & 0.1697 \\
\hline
\end{tabular}

${ }^{a} R_{1}=\Sigma|| F_{0}|-| F_{\mathrm{c}}|| / \Sigma\left|F_{0}\right|, w R_{2}=\left[\Sigma\left[w\left(F_{0}^{2}-F_{\mathrm{c}}^{2}\right)^{2}\right] / \Sigma\left[w\left(F_{0}^{2}\right)^{2}\right]\right]^{1 / 2}$

Table S2. The selected $\mathrm{Pb}-\mathrm{Cl}$ bond lengths $[\AA]$ and $\mathrm{Cl}-\mathrm{Pb}-\mathrm{Cl}$ bond angles $\left({ }^{\circ}\right)$ of $\mathbf{1}$ at $295 \mathrm{~K}$.

\begin{tabular}{c|c|c|c}
\hline $\mathrm{Pb}(1)-\mathrm{Cl}(1)$ & $\mathrm{Pb}(2)-\mathrm{Cl}(2)$ & $2.626(3)$ \\
$\mathrm{Pb}(1)-\mathrm{Cl}(1) \# 1$ & $2.867(3)$ & $\mathrm{Pb}(2)-\mathrm{Cl}(7)$ & $2.878(4)$ \\
$\mathrm{Pb}(1)-\mathrm{Cl}(3)$ & $2.880(3)$ & $\mathrm{Pb}(2)-\mathrm{Cl}(7) \# 4$ & $2.857(4)$ \\
$\mathrm{Pb}(1)-\mathrm{Cl}(3) \# 3$ & $3.039(4)$ & $\mathrm{Pb}(2)-\mathrm{Cl}(5)$ & $2.920(3)$ \\
$\mathrm{Pb}(1)-\mathrm{Cl}(6) \# 2$ & $2.971(3)$ & $\mathrm{Pb}(2)-\mathrm{Cl}(5) \# 5$ & $2.906(3)$ \\
$\mathrm{Pb}(1)-\mathrm{Cl}(6)$ & $2.785(4)$ & $\mathrm{Cl}(7)-\mathrm{Pb}(2) \# 7$ & $2.857(4)$ \\
$\mathrm{Cl}(3)-\mathrm{Pb}(1) \# 6$ & $3.039(4)$ & $\mathrm{Cl}(5)-\mathrm{Pb}(2) \# 8$ & $2.906(3)$ \\
$\mathrm{Cl}(6)-\mathrm{Pb}(1) \# 9$ & $2.971(3)$ & & \\
\hline $\mathrm{Cl}(6)-\mathrm{Pb}(1)-\mathrm{Cl}(1)$ & $88.79(9)$ & $\mathrm{Cl}(2)-\mathrm{Pb}(2)-\mathrm{Cl}(7) \# 4$ & $85.82(12)$ \\
$\mathrm{Cl}(6)-\mathrm{Pb}(1)-\mathrm{Cl}(1) \# 1$ & $88.79(9)$ & $\mathrm{Cl}(2)-\mathrm{Pb}(2)-\mathrm{Cl}(7)$ & $84.72(12)$ \\
$\mathrm{Cl}(1)-\mathrm{Pb}(1)-\mathrm{Cl}(1) \# 1$ & $164.63(15)$ & $\mathrm{Cl}(7) \# 4-\mathrm{Pb}(2)-\mathrm{Cl}(7)$ & $88.961(12)$ \\
$\mathrm{Cl}(6)-\mathrm{Pb}(1)-\mathrm{Cl}(3)$ & $85.12(12)$ & $\mathrm{Cl}(2)-\mathrm{Pb}(2)-\mathrm{Cl}(5) \# 5$ & $86.78(12)$ \\
$\mathrm{Cl}(1)-\mathrm{Pb}(1)-\mathrm{Cl}(3)$ & $82.33(7)$ & $\mathrm{Cl}(7) \# 4-\mathrm{Pb}(2)-\mathrm{Cl}(5) \# 5$ & $89.21(12)$ \\
$\mathrm{Cl}(1) \# 1-\mathrm{Pb}(1)-\mathrm{Cl}(3)$ & $82.33(7)$ & $\mathrm{Cl}(7)-\mathrm{Pb}(2)-\mathrm{Cl}(5) \# 5$ & $171.41(8)$ \\
$\mathrm{Cl}(6)-\mathrm{Pb}(1)-\mathrm{Cl}(6) \# 2$ & $93.16(4)$ & $\mathrm{Cl}(2)-\mathrm{Pb}(2)-\mathrm{Cl}(5)$ & $86.43(11)$ \\
$\mathrm{Cl}(1)-\mathrm{Pb}(1)-\mathrm{Cl}(6) \# 2$ & $97.65(7)$ & $\mathrm{Cl}(7) \# 4-\mathrm{Pb}(2)-\mathrm{Cl}(5)$ & $171.63(8)$ \\
$\mathrm{Cl}(1) \# 1-\mathrm{Pb}(1)-\mathrm{Cl}(6) \# 2$ & $97.65(7)$ & $\mathrm{Cl}(7)-\mathrm{Pb}(2)-\mathrm{Cl}(5)$ & $93.48(12)$ \\
$\mathrm{Cl}(3)-\mathrm{Pb}(1)-\mathrm{Cl}(6) \# 2$ & $178.27(12)$ & $\mathrm{Cl}(5) \# 5-\mathrm{Pb}(2)-\mathrm{Cl}(5)$ & $87.20(3)$ \\
$\mathrm{Cl}(6)-\mathrm{Pb}(1)-\mathrm{Cl}(3) \# 3$ & $171.53(11)$ & $\mathrm{Pb}(1)-\mathrm{Cl}(3)-\mathrm{Pb}(1) \# 6$ & $161.32(14)$ \\
$\mathrm{Cl}(1)-\mathrm{Pb}(1)-\mathrm{Cl}(3) \# 3$ & $90.08(9)$ & $\mathrm{Pb}(2) \# 7-\mathrm{Cl}(7)-\mathrm{Pb}(2)$ & $171.57(13)$ \\
$\mathrm{Cl}(1) \# 1-\mathrm{Pb}(1)-\mathrm{Cl}(3) \# 3$ & $90.08(9)$ & $\mathrm{Pb}(2) \# 8-\mathrm{Cl}(5)-\mathrm{Pb}(2)$ & $155.42(12)$ \\
$\mathrm{Cl}(3)-\mathrm{Pb}(1)-\mathrm{Cl}(3) \# 3$ & $86.41(2)$ & $\mathrm{Pb}(1)-\mathrm{Cl}(6)-\mathrm{Pb}(1) \# 9$ & $151.12(17)$ \\
$\mathrm{Cl}(6) \# 2-\mathrm{Pb}(1)-\mathrm{Cl}(3) \# 3$ & $95.32(11)$ & & \\
\hline$\# 1-\mathrm{x}+2, \mathrm{y}, \mathrm{z} ; \# 2-\mathrm{x}+2,-\mathrm{y}, \mathrm{z}+1 / 2 ; \# 3-\mathrm{x}+2,-\mathrm{y}+1, \mathrm{z}+1 / 2 ; \# 4 \mathrm{x},-\mathrm{y}+1, \mathrm{z}-1 / 2 ; \# 5 \mathrm{x},-\mathrm{y}, \mathrm{z}-1 / 2 ;$ \\
$\# 6-\mathrm{x}+2,-\mathrm{y}+1, \mathrm{z}-1 / 2 ; \# 7 \mathrm{x},-\mathrm{y}+1, \mathrm{z}+1 / 2 ; \# 8 \mathrm{x},-\mathrm{y}, \mathrm{z}+1 / 2 ; \# 9-\mathrm{x}+2,-\mathrm{y}, \mathrm{z}-1 / 2$ & \\
& & & \\
\hline & & & \\
\hline & & & \\
\hline
\end{tabular}




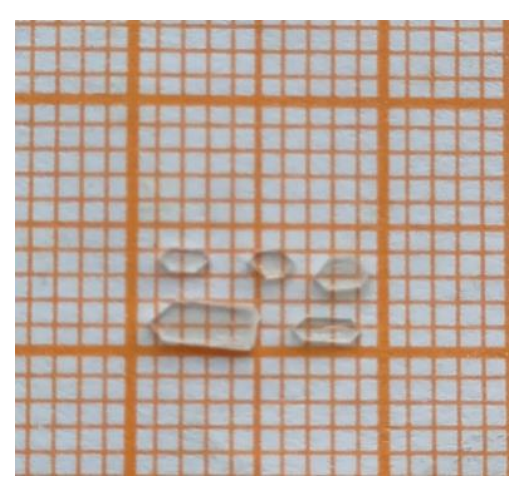

Figure S1. Block crystals of 1 .

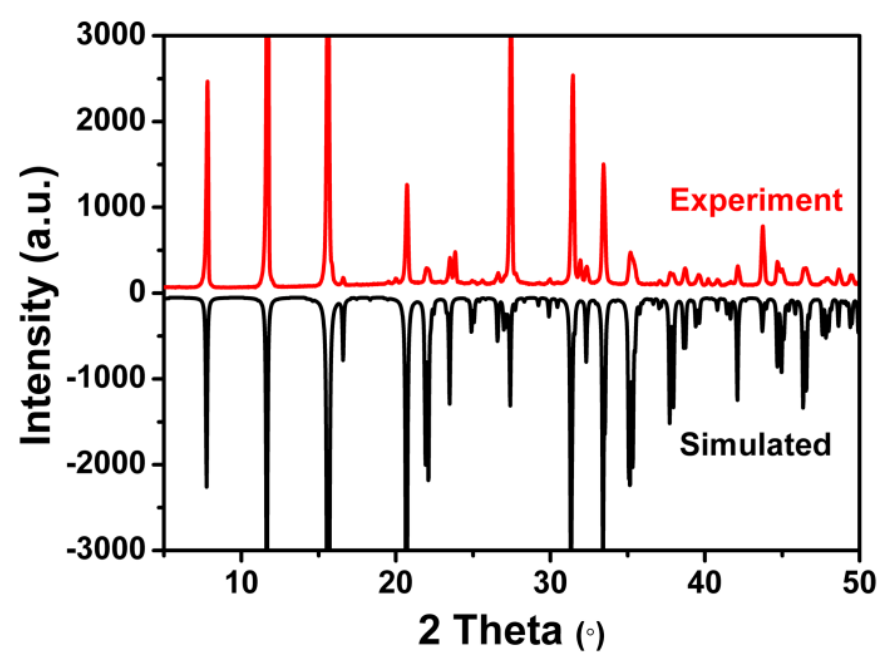

Figure S2. Experiment and simulated powder X-ray diffraction patterns of $\mathbf{1}$ at room temperature.

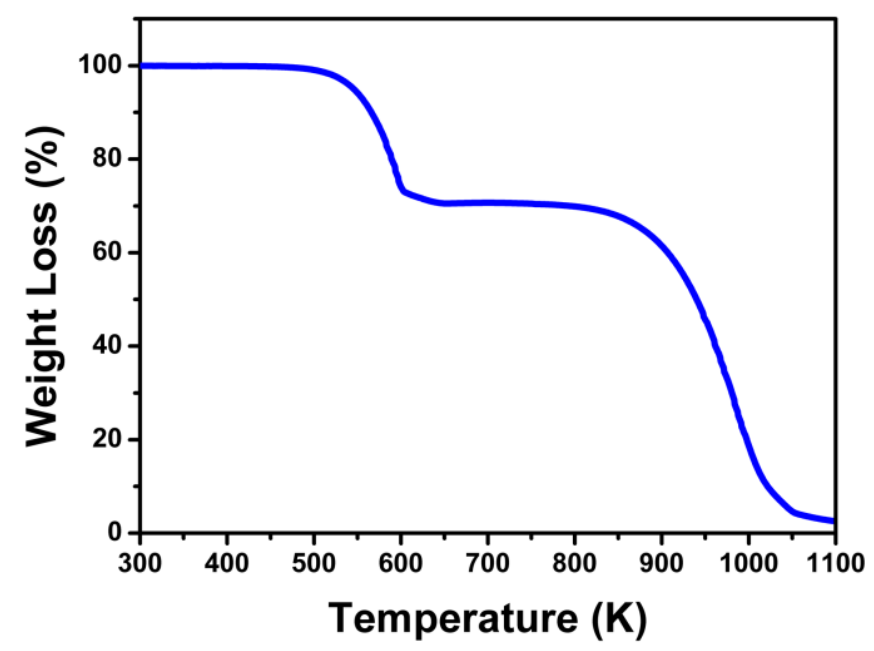

Figure S3. Thermogravimetric curve of 1 . 


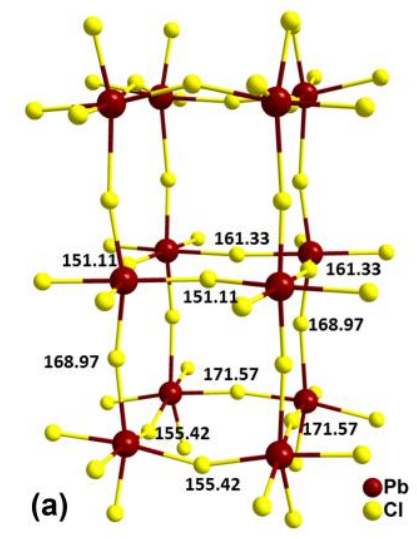

(b)

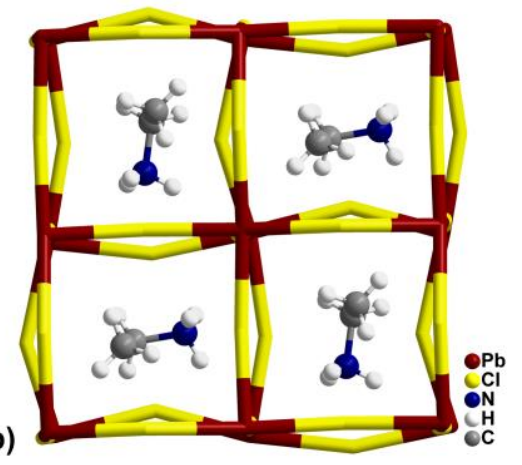

Figure S4. The selected $\mathrm{Pb}-\mathrm{Cl}-\mathrm{Pb}$ bond angels (a) and the distorted inorganic framework (b) of $\mathbf{1}$ at $295 \mathrm{~K}$.

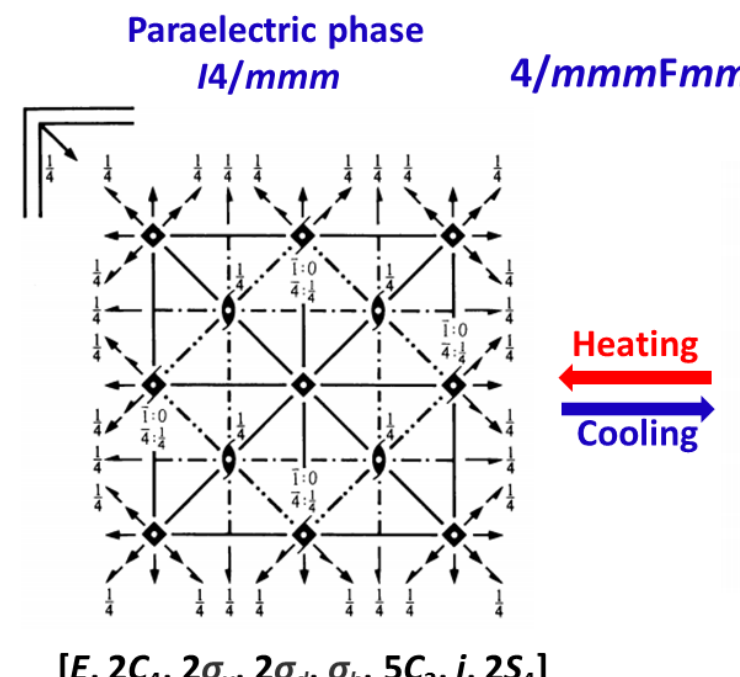

\section{Ferroelectric phase \\ $\mathrm{Cmc2}_{1}$}

$\left[E, 2 C_{4}, 2 \sigma_{v}, 2 \sigma_{d}, \sigma_{h}, 5 C_{2}, i, 2 S_{4}\right]$

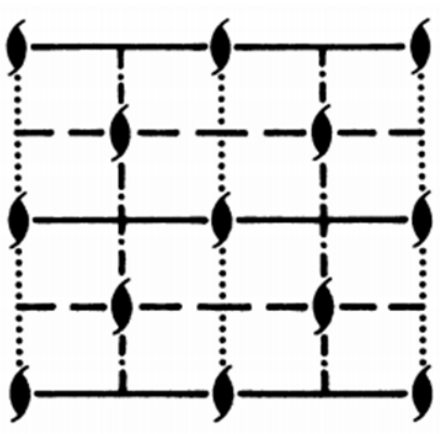

$\left[E, C_{2}, 2 \sigma_{v}\right]$

Figure S5. Symmetry breaking occurs in 1 with an Aizu notation of $4 / \mathrm{mmm}$ Fmm2.

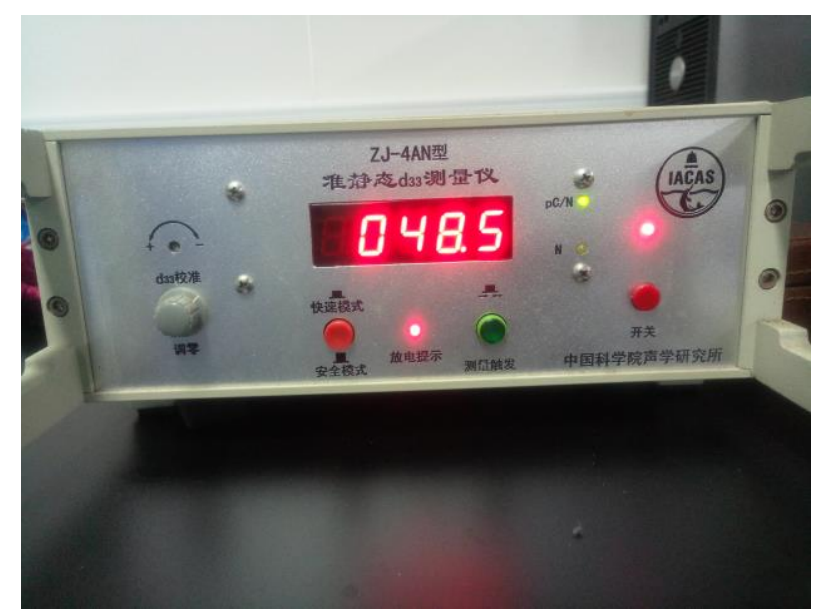

Figure S6. Photo of the piezoelectric coefficient $d_{33}$ of $\mathbf{1}$ measured along $c$-axis at room temperature. 


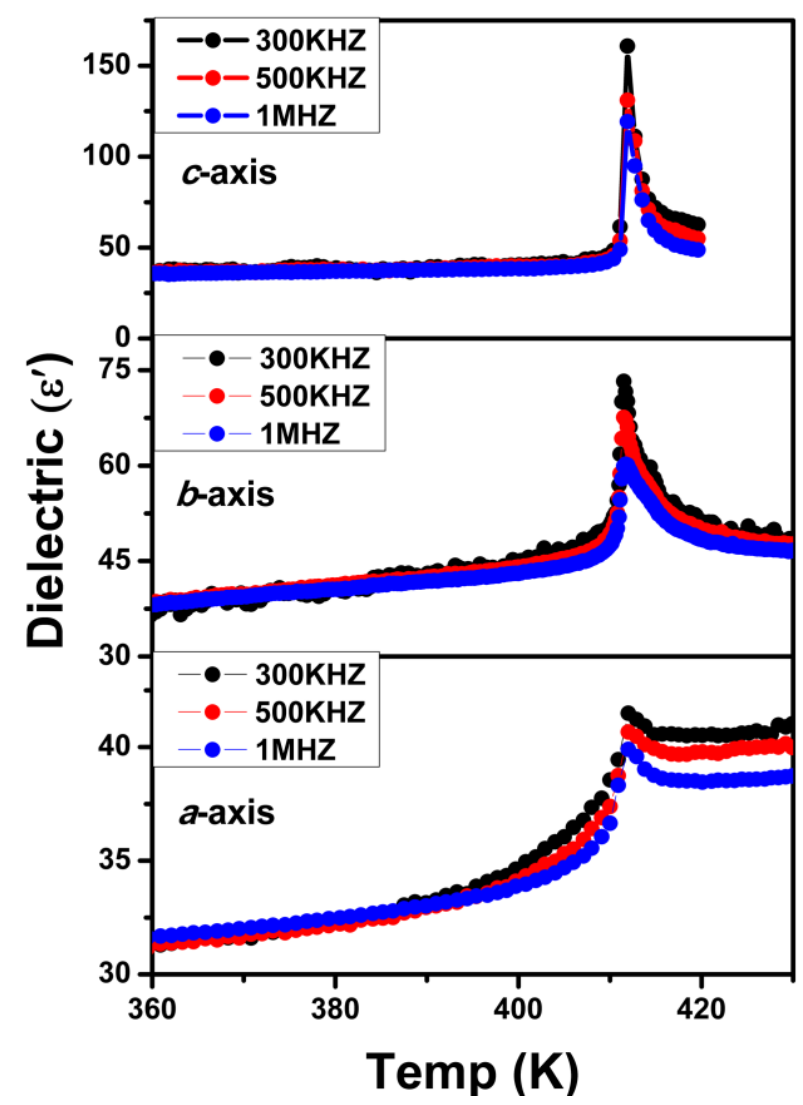

Figure S7. Variable-temperature dielectric permittivities $\left(\varepsilon^{\prime}\right)$ of $\mathbf{1}$ measured along the crystallographic $a$-, $b$-, and $c$-axes at different frequencies.

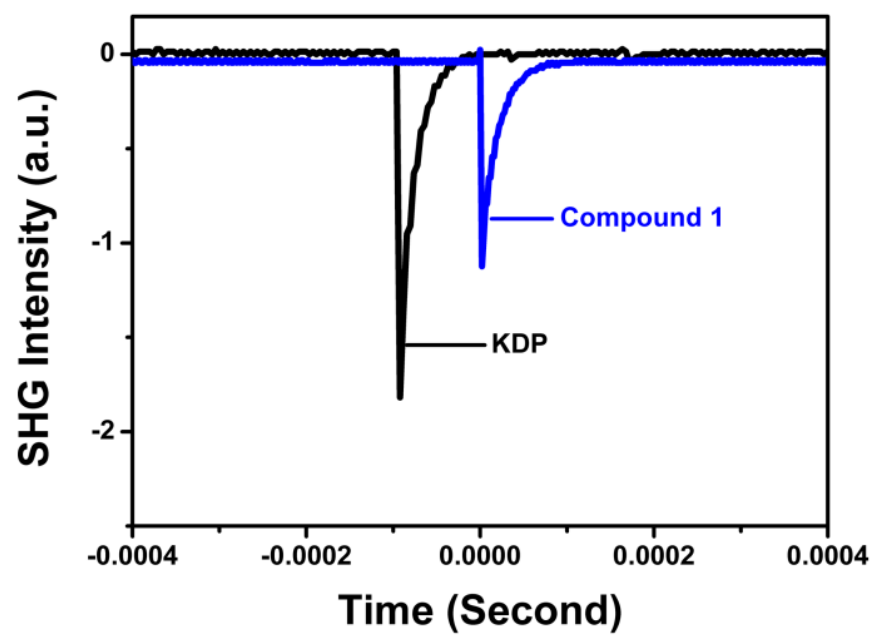

Figure S8. The SHG signals for the KDP standard and the polycrystalline sample of 1 at room temperature. 


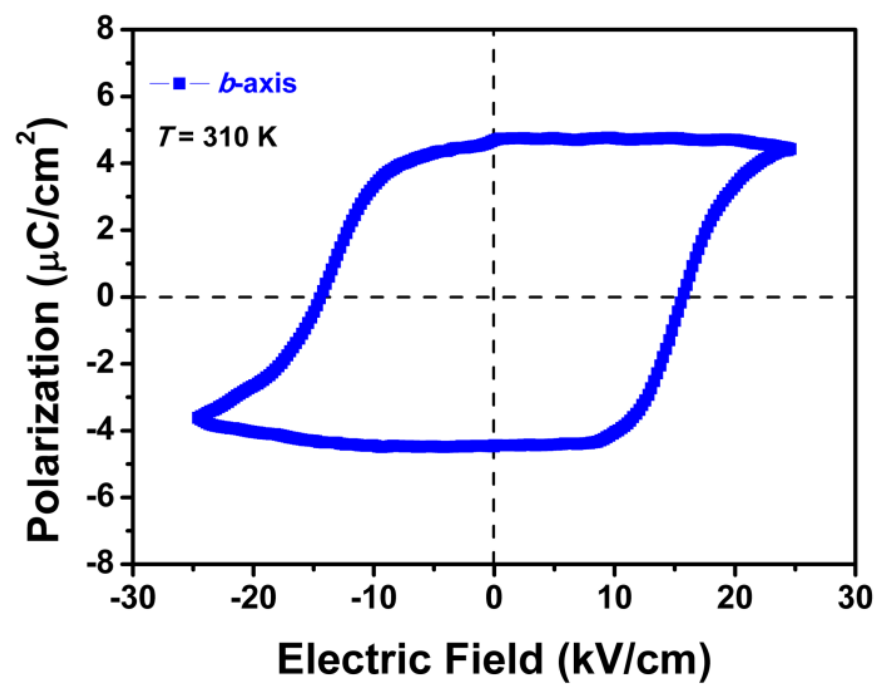

Figure S9. P-E hysteresis loop measured along the crystallographic $b$-axis at ferroelectric phase.

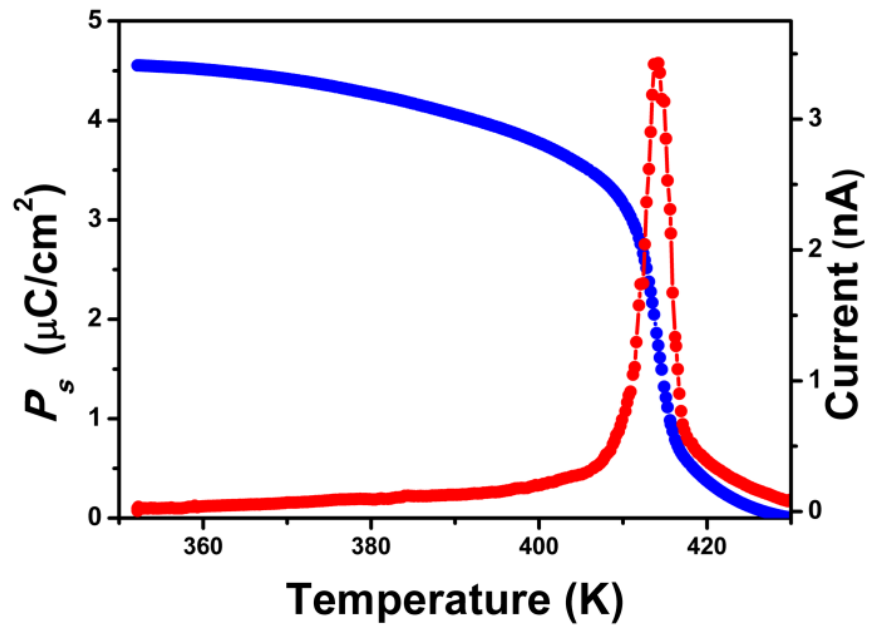

Figure S10. The temperature-dependent spontaneous polarization $\left(P_{s}\right)$ of $\mathbf{1}$ obtained by integrating the pyroelectric current.

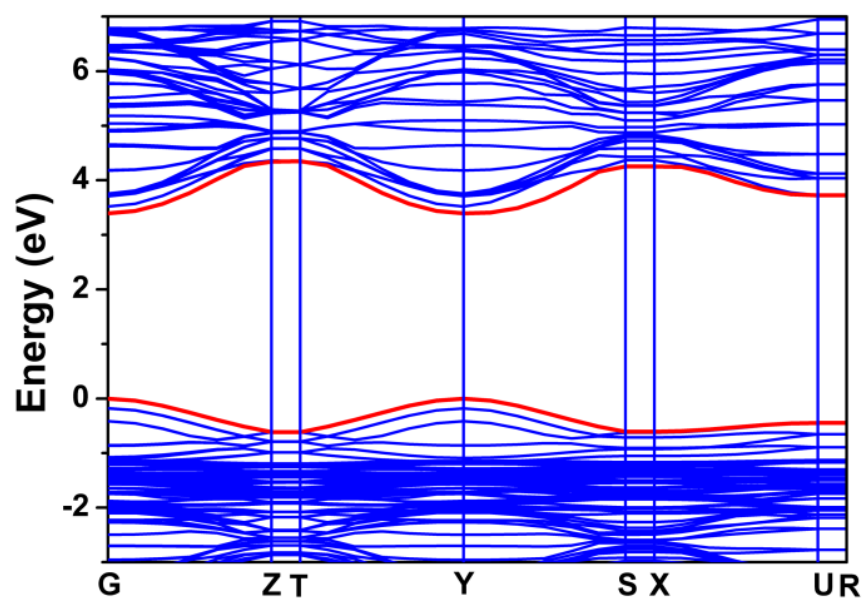

Figure S11. The calculated band structure of 1. 


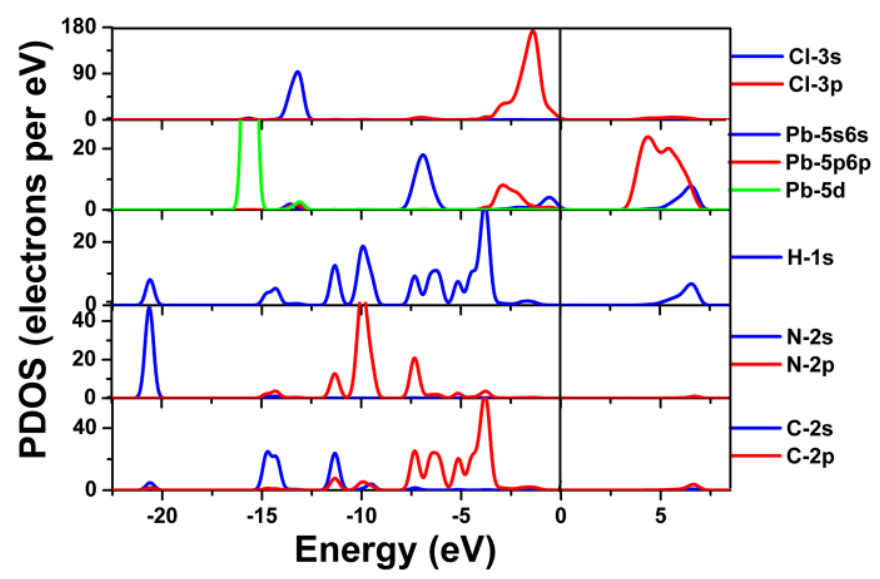

Figure S12. The partial density of states of $\mathbf{1}$.

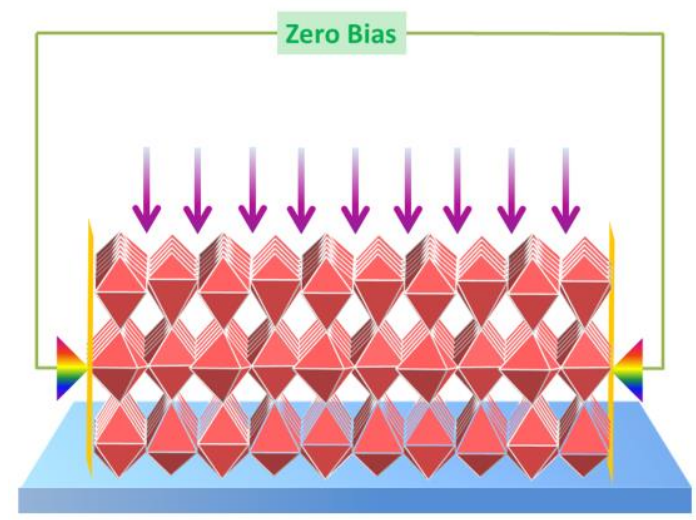

Figure S13. Schematic diagram of the device structure of 1.

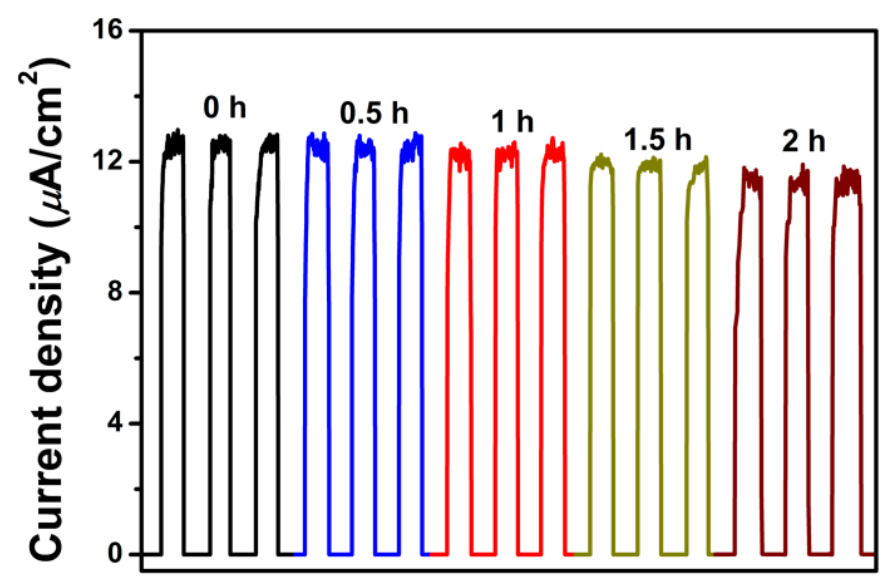

Figure S14. On/off switches for different hours exposure to ambient condition. 


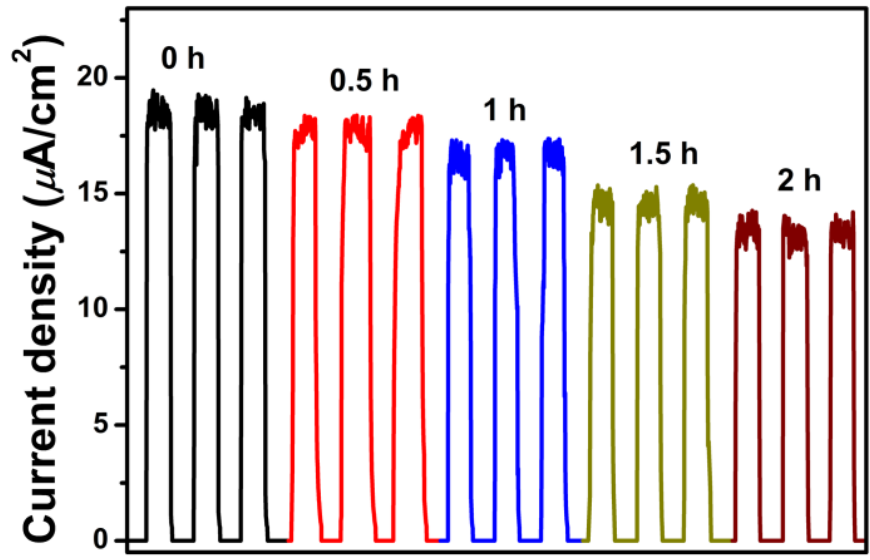

Figure S15. On/off switches for different hours exposure to ambient condition after polarization.

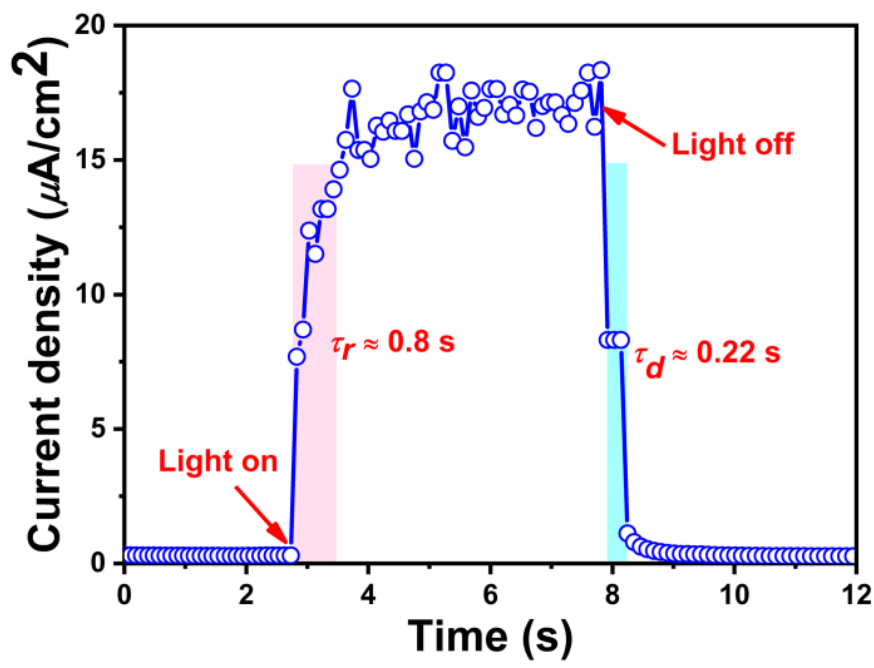

Figure S16. The rise and decay of photocurrent responses of $\mathbf{1}$ at zero-bias.

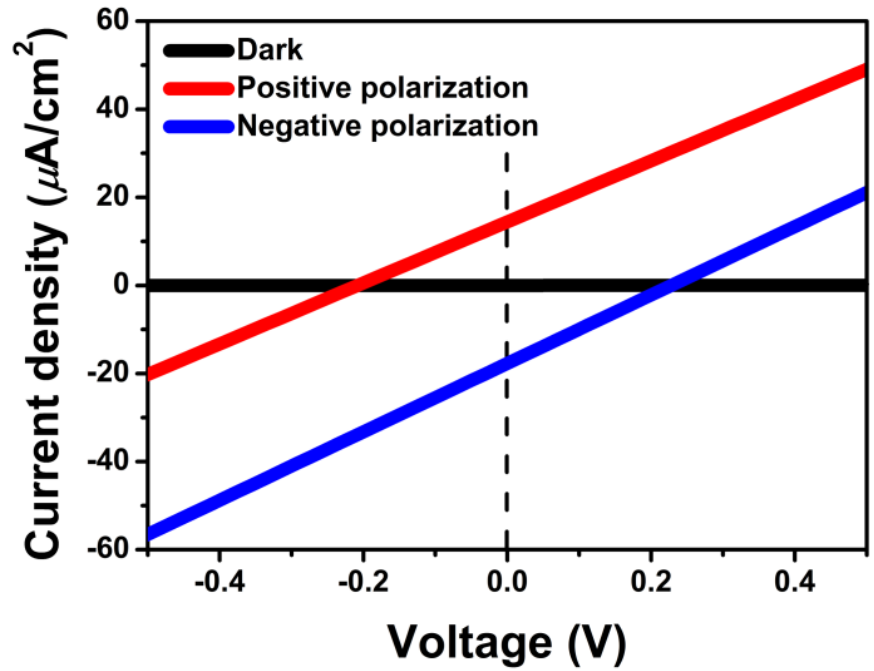

Figure S17. Switchable behaviors of bulk photovoltaic effects of 1 measured along $b$-axis. 


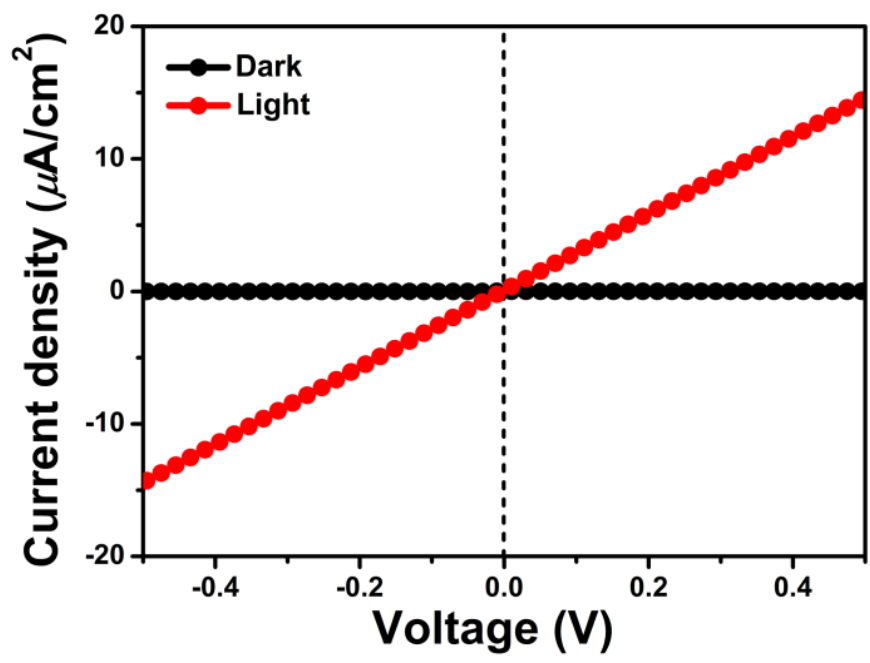

Figure S18. Current-Voltage characters of 1 in the dark and under illumination measured along $a$-axis. 